\title{
ANKLE-KNEE REHABILITATION SYSTEM
}

\author{
Amin S Abdullah Abdullah ${ }^{1}$, Cristian Gabriel Alionte ${ }^{2}$ and Constantin Nitu ${ }^{3}$ \\ 1,2,3 University "POLITEHNICA" of Bucharest \\ 313, Spl. Independentei, Bucharest-060042, Romania, \\ E-mail: almuhandes1979@yahoo.com, cristian.alionte@upb.ro
}

\begin{abstract}
In this paper is present a new type of a rehabilitation system for both knee and ankle that integrate two treatment types. The system allows a continuous passive motion consisting of two rotations of the leg segments, one of the knee articulation and one of the ankle joint, allowing the functional rehabilitation of the individuals that have an impairment of walking due to a disease or an accident. This is one novelty of the system because all the system that are on the market now consider to one articulation that can be knee or the ankle. In addition, the system allows the application of a mechanical vibration at the lower level of the leg, which have a beneficial action on the bones, muscle, and nerves, which is a well-known treatment.
\end{abstract}

Keywords: Vibration Rehabilitation System, Functional Rehabilitation, Modelling, FEM Analysis.

\section{Introduction}

It is well known that, for the realization of a mechatronic gait simulation system must be considered several input parameters those which are as closest possible with the real ones owned by the patient. The parameters can be obtained from measuring using non-invasive methods, usually optical ones [1], because these parameters are individualized for each individual due to various factors:

- Body features: height and weight;

- Anatomy of the person: length of body segments, muscle tone;

- Psychosomatic features: personality, activism, type of life (sedentary, sporting), etc.;

- The gait ontogenesis because each person during childhood acquire two-leg and gait body stability mechanisms using individualized systems and methods.

- Gait mechanism is into a continuously transformation which takes place throughout the life span.

- Gait parameters usually are obtain using anthropogenesis measurement as function of averaging different actual measurement and therefore it cannot be said that gait is correct unless it adversely affects other components of the human body including gait.

Using the before mentioned facts can be conclude that when it is desired to develop a medical rehabilitation system for walking, it is important to consider the individual kinetic parameters of the patient.
These can be done through the development and implementation of a simpler walking analysis system that can be adapted to any patient and does not lead to a change in the usual course of the patient. In literature are several studies regarding human gait devices starting from Leonardo da Vinci, Galileo, Lagrange and Bernoulli [2] that have an interest in human gait until now. One of the first modern devices for gait assistance was developed by Hughes [3], Townsend [5] and Vukobratovic [4] and exist more sophisticated means for human help [6].

In this articleis presented a system, which was developed within the framework of the dissertation thesis of Mr. Abdullah Amin S. Abdullah [7], which aimed at researching the design and realization of a biomechanical system for the medical rehabilitation of the inferior limb by a continuous passive movement of the foot. Consideration has been given to balancing several factors in the design process, including cost and simplicity of design.

\section{The Rehabilitation System}

Continuous passive motion mechanisms are used for long-term by persons, which are bed immobilized, or after surgery or during the early postoperative period. Continuous passive motion mechanisms are available for many joints of the body, knee, ankle, jaw, hip, elbow, shoulder and finger joints. The study, presented in this article, analyses ensemble kneeankle only. 


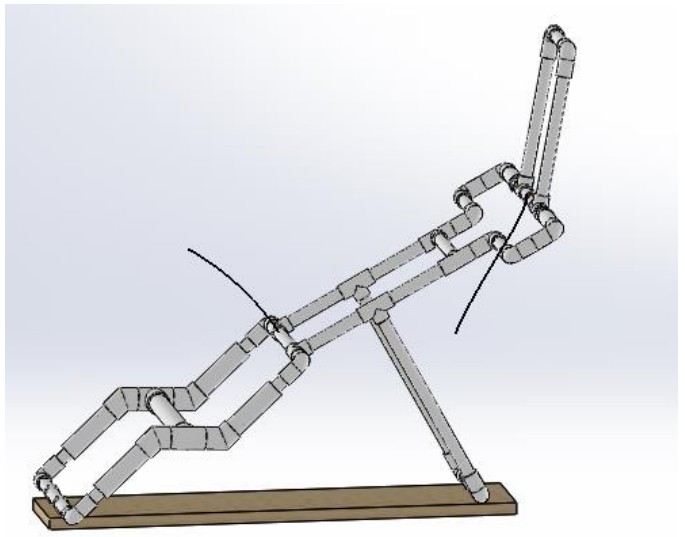

Figure 1: The 3D model of the medical rehabilitation system of ankle and knee.

A continuous passive motion mechanism was designed with six mobile elements and eight joints as can be seen in Fig.1. The continuous passive motion mechanism used two motors, one motor for knee motion and another for ankle.

The action of the foot was realized by combined motion. Two elements can be adapted to variable length, so the study presents the mathematical model for each position.The passive motion was used to prevent ankle contracture and the vibration to lessen muscle tone, to strengthen muscle power and to prevent thrombosis.The system has three independent systems:

- system 1 for the movement tibia and fibula which will produce the rotation of the knee;

- system 2 for the movement of the forefoot which will produce the rotation of the ankle;

- system 3 for vibration excitation of the muscles: soleus, gastrocnemius, popliteus, plantaris, flexor digitorum longus, flexor halluces longus and tibialis posteriorus and bones the tibia and fibula.

The first system consists of one stepper motor, which is moving through a belt a frame on which the leg is fastened. The motor is a stepper one so the control is very easy to implement. The motion is transmitted to an L-frame, which has a linear guide on the bottom and is connected on a parallelogram system in the upper part. Also, this frame is support the system 2 which only have a rotational movement of the patient foot. The movement is generated using a second stepper motor. The vibration is produced using an exciter at the upper level of the L-frame and is transmitted through the structure to the muscles and bones of the leg.

\section{Modeling and Simulation}

Within the project we have developed a continuous rehabilitation medical structure consisting of six mobile elements and eight joints. We used two engines to move the structure, one at the knee and one at the heel that will generate a combined motion.
The degree of mobility of the whole system can be described by Equation 1:

$M=6 n-\sum_{m=1}^{5} m * C_{m}$

Where $\mathrm{n}$ represents the number of mobile elements, $\mathrm{Cm}$ is the number of pair elements with $\mathrm{m}$ constraints. The degree of mobility highlights the number of independent elements that define the status parameters of the mechanism. It will be considered two distinct solutions, one at the joint $\mathrm{A}$ and one at the $\mathrm{G}$ joint (Figure 1).

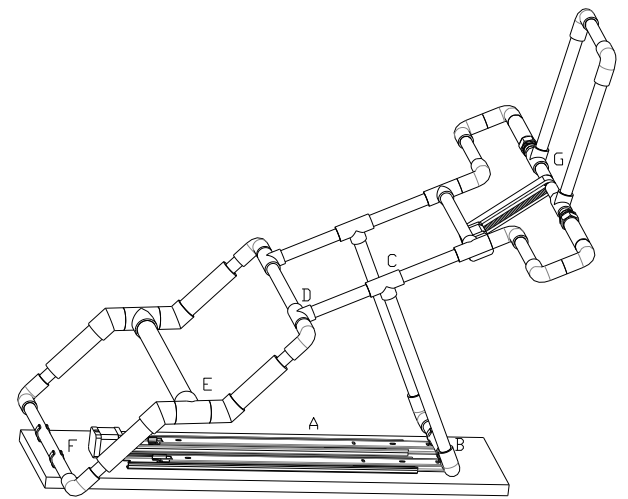

Figure 2: Thejoints and elements of the medical rehabilitation system.

There are 2 elements DE and EF to adjust the length of the D-joint so we need to study the mathematical model that takes into account the two positions. Points G, E, C are chinking. We will identify vectors of the contour line positions associated with the kinematic chain, i.e., ABCDEFA, whichever is more oriented and positioned as far as possible from the point of view of the angle. These contour lines can be rewritten as vector:

$\overline{A B}+\overline{B C}+\overline{C D}=\overline{D E}+\overline{E F}+\overline{F A}$

The knee mathematical model is based on scalar equations obtained from vector equations. For a geometric and kinematic analysis, the following dimensions of the elements were considered. $\mathrm{AB}=0.079[\mathrm{~m}] ; \quad \mathrm{BC}=0.328[\mathrm{~m}] ; \quad \mathrm{CD}=0.162[\mathrm{~m}]$; $\mathrm{DE}=0.244[\mathrm{~m}] ; \mathrm{EF}=0.187[\mathrm{~m}] ; \mathrm{FA}=0.3 \ldots 0.047[\mathrm{~m}]$.

To determine equation solutions, the PTC Mathcad Express Free [8] program was used based on a congruent program was used based on a congruent approximation method and the least squares method to solve the computational system.

Several values were given for angular displacements, but only those values for which they allow continuous movement were selected. In Fig 3. are presented the simulations results at the $\mathrm{D}$ joint (knee level) and at G Joint (ankle level) considering the displacement at the knee level.

The simulation is taken into account the origin of the coordinate system that is at F joint. 


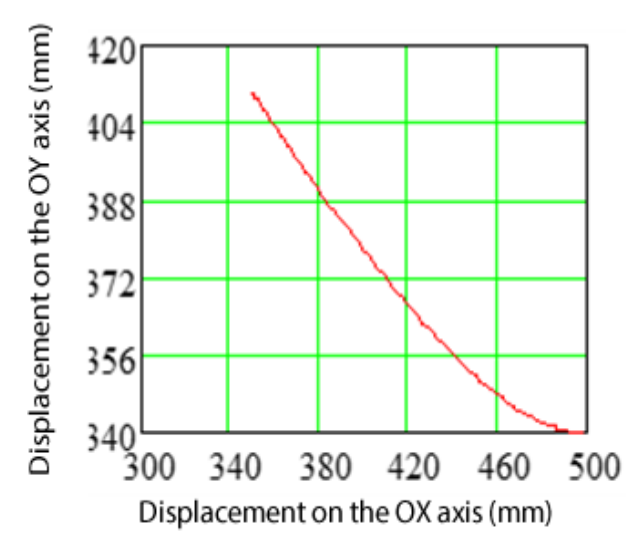

a)

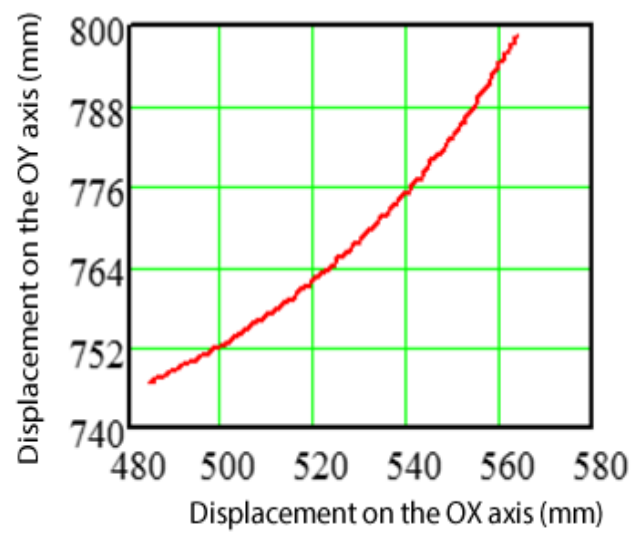

b)

Figure 3: Thejoints and elements of the medical rehabilitation system

\section{Results and Conclusions}

The first analyses are take into account only the mechanical stresses that actioned on the leg support bars independently. The first simulation is done with the support platform fixed and a force of $10 \mathrm{~N}$ which is acting on the ankle sustain bar. The system has a minimal deformation of $0.006 \mathrm{~mm}$ and a maximum stress on the 1 st frame vertical bars.

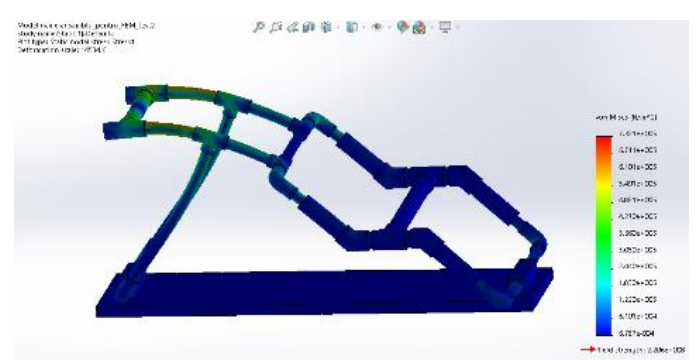

(a)

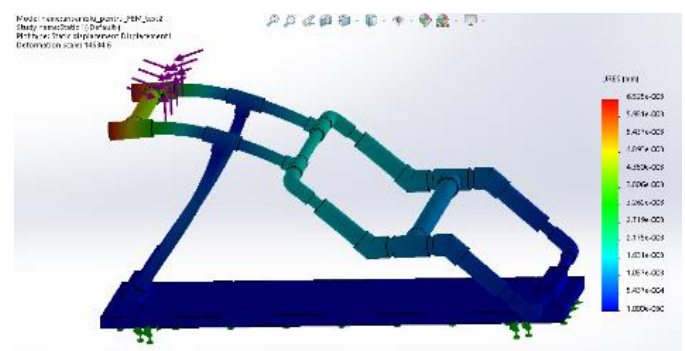

(b)

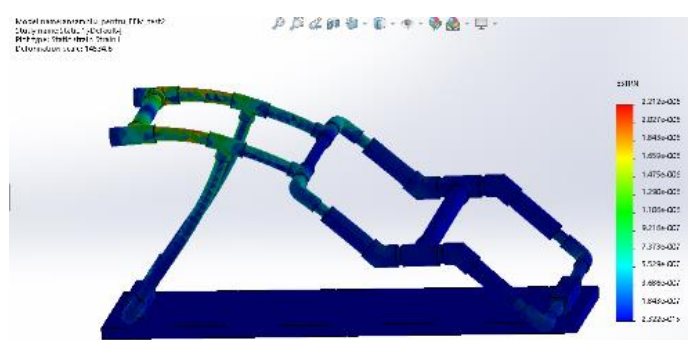

(c)

Figure 4: FEM analysis results for a mechanical force of $10 \mathrm{~N}$ on the ankle support bar:

(a)mechanical stress (b) deformation and (c) strain.
The second simulation is done with the support platform fixed and a force of $10 \mathrm{~N}$ which is acting on the knee sustain bar.

The system has a minimal deformation of 0.0017 $\mathrm{mm}$ and a maximum stress on the 1 st frame vertical bars and on the 2 nd frame.

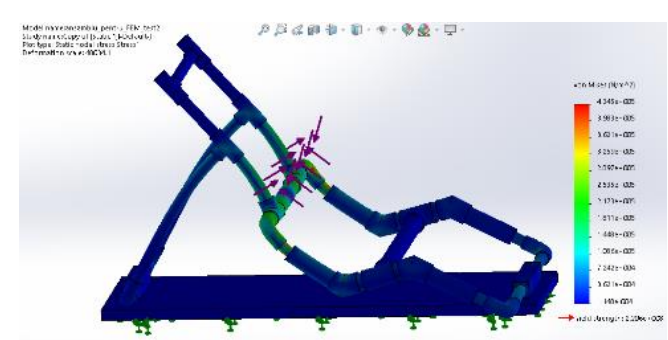

(a)

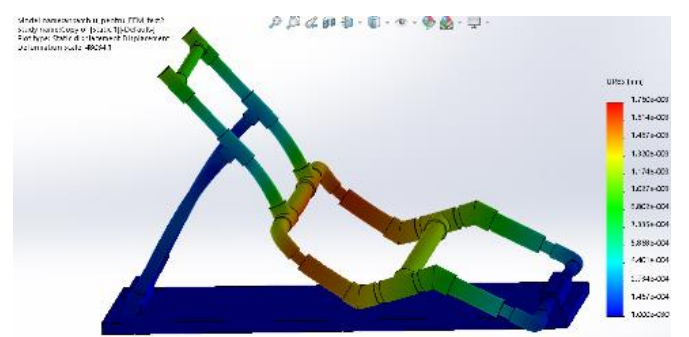

(b)

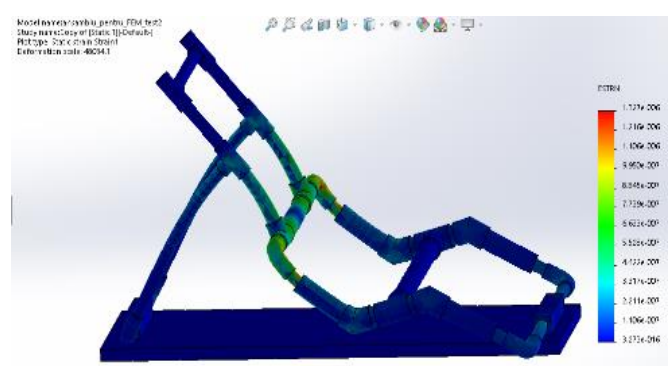

(c)

Figure 5: FEM analysis results for a mechanical force of $10 \mathrm{~N}$ on the knee support bar:

(a)mechanical stress b) deformation and c) strain. 
The third simulation is done with the support platform fixed and a force of $10 \mathrm{~N}$ which is acting on the thigh sustain bar. The system has a minimal deformation of $0.09 \mathrm{~mm}$ and a maximum stress on the 2 nd frame.

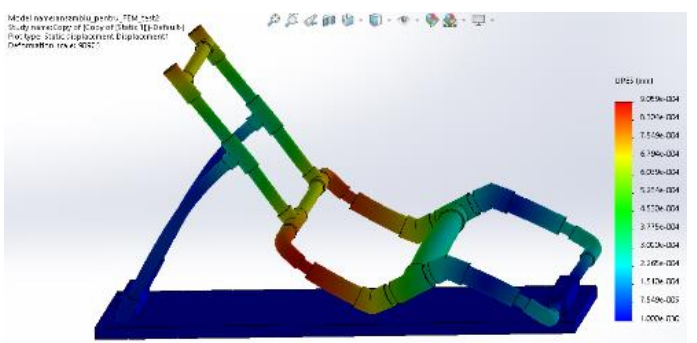

(a)

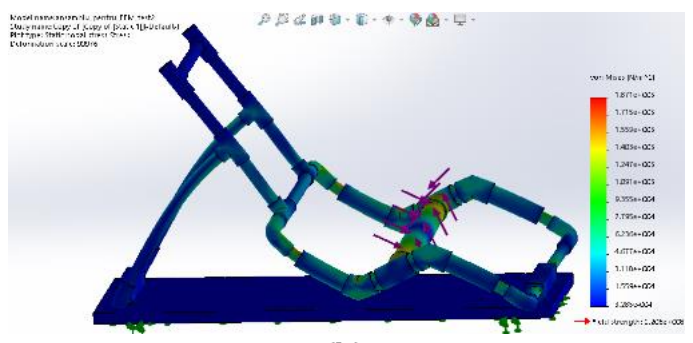

(b)

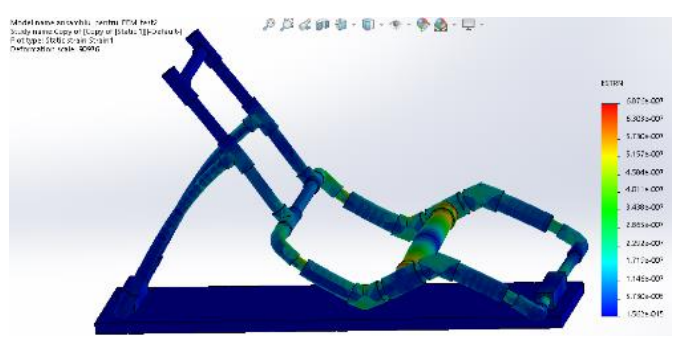

(c)

Figure 6: FEM analysis results for a mechanical force of $10 \mathrm{~N}$ on the thigh support bar

(a)mechanical stress (b) deformation and (c) strain.

The forth simulation is done with the support platform fixed and a force of $10 \mathrm{~N}$ which is acting on all three sustain bar. The system has a minimal deformation of $0.014 \mathrm{~mm}$ and a maximum stress on the 2 nd frame.

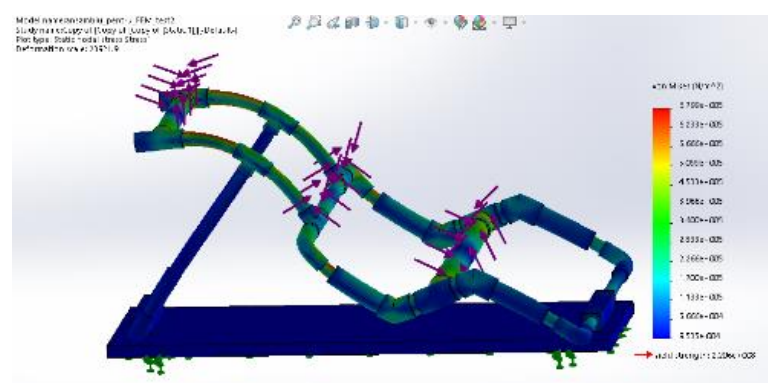

(a)

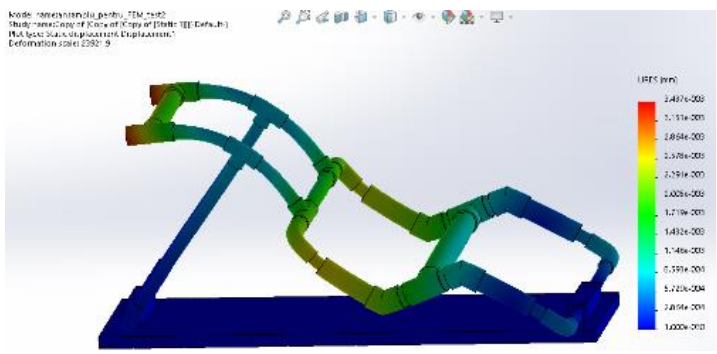

(b)

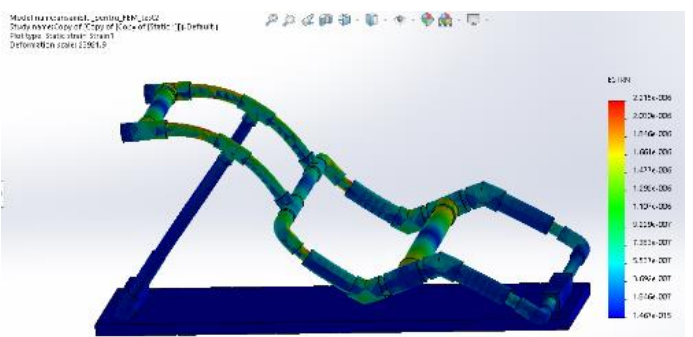

(c)

Figure 7: FEM analysis results for a mechanical force of $10 \mathrm{~N}$ on all three support bar:

(a)mechanical stress b) deformation c) and strain.

Continuous passive motion mechanism in combination with vibration reduced the muscle tone in the shank muscles and seem to be a safe and a feasible method to support ankle mobilization.

Therefore it was made a modal frequency analysis to determine the natural frequency and 1st, 2nd ,3rd 4th and 5th harmonics:

- the natural frequency is at $61.276 \mathrm{~Hz}$ (the structure oscillates as in figure 8a);

- the second frequency is at $117.21 \mathrm{~Hz}$ (the structure oscillates as in figure $8 \mathrm{~b}$ );

- third frequency is at $132.05 \mathrm{~Hz}$ (the structure oscillates as in figure 8c);

- the forth frequency is at $156.36 \mathrm{~Hz}$ (the structure oscillates as in figure 8d);

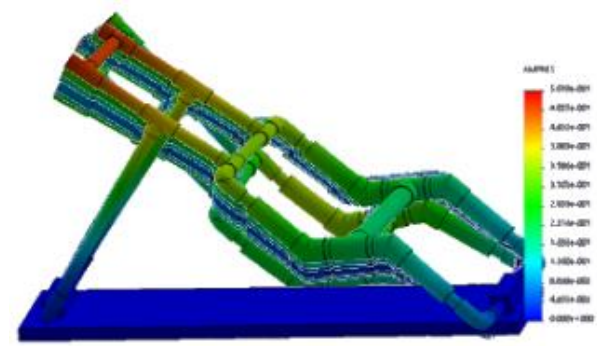

(a)

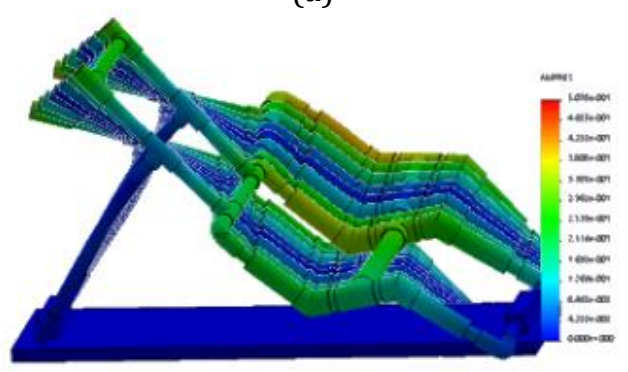

(b) 


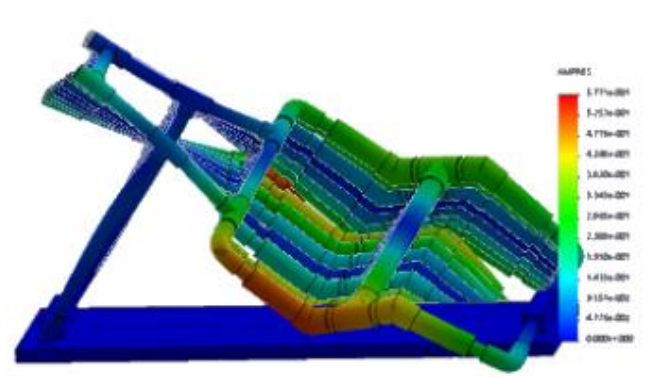

(c)

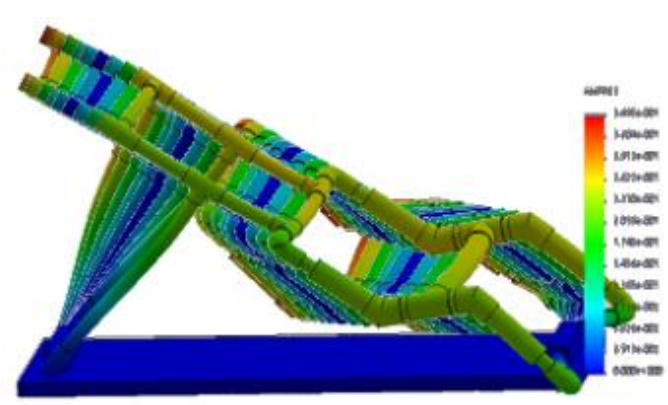

(d)

Figure 8: Modal analysis:

(a) the vibration of the structure at the frequency of $61.276 \mathrm{~Hz}$; (b) the vibration of the structure at the frequency of $117.21 \mathrm{~Hz}$; (c) the vibration of the structure at the frequency of $132.05 \mathrm{~Hz}$;

(d) the vibration of the structure at the frequency of $61.276 \mathrm{~Hz}$.

\section{References}

[1] Sturman, D. and Zeltzer, D.: A survey of glove based input. In: IEEE Computer Graphics and Aplications, 30-39(1994).

[2] Winter, D. A.: Biomechanics and Motor Control of Human Movement. John Wiley \& Sons, Canada(2009).

[3] Hughes, J.: Powered lower limb orthotics in paraplegia.In: Paraplegia,191-193 (1972)

[4] Townsend, M. A.: Dynamics and coordination of torso motion in human locomotion. In: Journal Biomechanics, 727-738(1981).

[5] Vukobratovic, M. and Borovac, B.: Zero-Moment Point- thirty five years of its life. In: International Journal of Humanoid Robotics, Vol. 1, No. 1, 157 173(2004).

[6] Banala, S. K. and Agrawal, S.: Gait rehabilitation with an active leg orthosis. In: Proceedings ASME Mechanisms and Robotics Conference(2005)

[7] Abdullah A.S.A.: Design and construction of a biomechanical system for medical rehabilitation. Msc. Dissertation Precision Mechanics, University "Politehnica" of Bucharest (2017)

[8] PTC Mathcad Homepage, https://www.ptc.com/en/products/mathcadexpress-free-download,last accessed $2017 / 10 / 14$. 